\title{
UNBOUNDED MULTIPLIERS ON OPERATOR SPACES
}

\author{
HENDRIK SCHLIETER AND WEND WERNER
}

(Communicated by Marius Junge)

\begin{abstract}
We introduce unbounded multipliers on operator spaces. These multipliers generalize both regular operators on Hilbert $C^{*}$-modules and (bounded) multipliers on operator spaces.
\end{abstract}

\section{INTRODUCTION}

Unbounded multipliers play an important role in mathematics, for example in the spectral theorem for unbounded self-adjoint operators. In the context of Hilbert $C^{*}$-modules, unbounded multipliers are called regular operators. The definition of such an operator goes back to A. Connes, Con81, who defines the notion of a self-adjoint regular operator on a $C^{*}$-algebra. S. L. Woronowicz defines in Wor91] general regular operators on $C^{*}$-algebras. Regular operators are important in the theory of noncompact quantum groups (Wor91, [WN92]) and can be used to define unbounded Kasparov modules in Kasparov's bivariant $K K$-theory ([BJ83]).

In this article we define unbounded multipliers on operator spaces. On a Hilbert $C^{*}$-module, equipped with a canonical operator space structure, these multipliers coincide with the regular operators. Moreover, every bounded left adjointable multiplier on an operator space belongs to the class of unbounded multipliers.

We will give several characterizations of unbounded multipliers on operator spaces. For example, it turns out that, roughly, every unbounded multiplier on an operator space is the restriction of a regular operator and that an unbounded multiplier can be characterized using the theory of strongly continuous groups on a Hilbert space. As an application we will generalize a pertubation result for regular operators on Hilbert $C^{*}$-modules to operator spaces.

Most of the results of this paper are from [Sch09].

\section{Preliminaries}

For the basic theory of $C_{0}$-semigroups, we refer the reader to EN00. Recall that the generator of a $C_{0}$-semigroup is unique, densely defined and closed.

2.1. Regular operators. We begin by recalling some basic facts about regular operators. For more details, we refer to Wor91, Lan95] and Kus97.

Let $E, F$ be Hilbert $C^{*}$-modules over a $C^{*}$-algebra $\mathfrak{A}$.

Received by the editors July 22, 2010 and, in revised form, September 11, 2011.

2010 Mathematics Subject Classification. Primary 46L07; Secondary 46L08, 47D06.

Key words and phrases. Operator spaces, $C^{*}$-modules, unbounded multipliers.

The first author was partially supported by the SFB 478 - Geometrische Strukturen in der Mathematik at the Westfälische Wilhelms-Universität Münster, supported by the Deutsche Forschungsgemeinschaft. 
Definition 2.1. Let $A: D(A) \subseteq E \rightarrow F$ be a densely defined, A-linear map where $D(A)$ denotes the domain of $A$. Then

$$
D\left(A^{*}\right):=\left\{y \in F \mid \exists z_{y} \in E \forall x \in D(A):\langle A x, y\rangle=\left\langle x, z_{y}\right\rangle\right\}
$$

is a submodule of $F$. For all $y \in D\left(A^{*}\right)$ the element $z_{y}$ is uniquely defined and denoted by $A^{*} y$. We get an $\mathfrak{A}$-linear, closed map $A^{*}: D\left(A^{*}\right) \subseteq F \rightarrow E$, called the adjoint of $A$, satisfying $\left\langle x, A^{*} y\right\rangle=\langle A x, y\rangle$ for all $x \in D(A)$ and $y \in D\left(A^{*}\right)$.

Definition 2.2. A regular operator is a densely defined, closed, $\mathfrak{A}$-linear map $A: D(A) \subseteq E \rightarrow F$ such that $A^{*}$ is densely defined and $1+A^{*} A$ has dense image. The set of regular operators is denoted by $\mathcal{R}(E, F)$. Define $\mathcal{R}(E):=\mathcal{R}(E, E)$.

Regular operators are a generalization of densely defined, closed operators on a Hilbert space $H$, because the set of regular operators on $H$ is equal to the set of densely defined, closed operators on $H$. Denote by $\mathbb{B}(E)$ the set of adjointable operators on $E$. Then

$$
\mathbb{B}(E)=\{A \in \mathcal{R}(E) \mid D(A)=E\} .
$$

If $\mathfrak{A}$ is a unital $C^{*}$-algebra, we get $\mathcal{R}(\mathfrak{A})=\mathfrak{A}$.

A regular operator $A \in \mathcal{R}(E)$ is called self-adjoint (resp. skew-adjoint) if $A^{*}=A$ (resp. $A^{*}=-A$ ). For every regular operator $A \in \mathcal{R}(E, F)$ we can define the adjointable operator $z_{A}:=A\left(\left(1+A^{*} A\right)^{-1}\right)^{1 / 2}$ with $\left\|z_{A}\right\| \leq 1$. We denote by $C(\mathbb{R})$ the set of continuous functions from $\mathbb{R}$ to $\mathbb{C}$.

If $A \in \mathbb{B}(E)$ we already have the notion of a functional calculus and spectrum for $A$, because $\mathbb{B}(E)$ is a $C^{*}$-algebra. For self-adjoint regular operators we have the following functional calculus:

Theorem 2.3 ([Lan95, Theorem 10.9]). Let $A \in \mathcal{R}(E)$ be self-adjoint. Let $\iota$ be the canonical inclusion of $\mathbb{R}$ into $\mathbb{C}$ and $f: \mathbb{R} \rightarrow \mathbb{C}, \lambda \mapsto \lambda\left(1+\lambda^{2}\right)^{-1 / 2}$. Then there exists a unique ${ }^{*}$-homomorphism $\varphi_{A}: C(\mathbb{R}) \rightarrow \mathcal{R}(E)$ such that $\varphi_{A}(\iota)=A$ and $\varphi_{A}(f)=z_{A}$.

Woronowicz introduced the following consistent definition:

Definition 2.4. Let $A \in \mathcal{R}(E)$ be self-adjoint. The set

$$
\sigma(A):=\left\{\lambda \in \mathbb{R} \mid \forall f \in \operatorname{ker}\left(\varphi_{A}\right): f(\lambda)=0\right\}
$$

is called the spectrum of $A$.

The set $\sigma(A)$ is a closed, nonempty subset of $\mathbb{R}$.

2.2. Stone's theorem for Hilbert $C^{*}$-modules. Let $E$ be a Hilbert $C^{*}$-module over a $C^{*}$-algebra $\mathfrak{A}$. The following two results are generalizations of HQVK92, Theorem 2.1 and Proposition 2.2] from $C^{*}$-algebras to Hilbert $C^{*}$-modules; see also WN92, Theorem 2.3]. With some adjustments the proof of the first statement can be transferred to the Hilbert $C^{*}$-module case. For details see [Sch09], Proposition 1.27 and Satz 1.30.

Theorem 2.5. Let $A \in \mathcal{R}(E)$ be self-adjoint. Let $e_{t}: \mathbb{R} \rightarrow \mathbb{C}, \lambda \mapsto \exp (\mathrm{i} \lambda t)$, and $U_{t}:=\exp (\mathrm{i} t A):=\varphi_{A}\left(e_{t}\right)$ for all $t \in \mathbb{R}$. Then $\left(U_{t}\right)_{t \in \mathbb{R}}$ is a $C_{0}$-group on $E$ with generator $\mathrm{i} A$ such that $U_{t}$ is a unitary element of $\mathbb{B}(E)$ for all $t \in \mathbb{R}$. 
Theorem 2.6 (Stone's theorem). Let $\left(U_{t}\right)_{t \in \mathbb{R}}$ be a $C_{0}$-group on $E$ such that $U_{t}$ is a unitary element of $\mathbb{B}(E)$ for all $t \in \mathbb{R}$.

(i) There exists a self-adjoint $A \in \mathcal{R}(E)$ such that $U_{t}=\exp (\mathrm{i} t A)$ for all $t \in \mathbb{R}$.

(ii) If $\left(U_{t}\right)_{t \in \mathbb{R}}$ is norm continuous, then $A \in \mathbb{B}(E)$.

Proof. To prove (i) denote by $C^{*}(\mathbb{R})$ the group $C^{*}$-algebra of the locally compact group $\mathbb{R}$ and by $\hat{\mathbb{R}}$ the dual group of $\mathbb{R}$. We have $\hat{\mathbb{R}} \cong \mathbb{R}$. Let $C_{0}(\mathbb{R})\left(\operatorname{resp} . C_{c}(\mathbb{R})\right)$ denote the set of continuous functions on $\mathbb{R}$ vanishing at infinity (resp. with compact support). For all $f \in C_{c}(\mathbb{R})$,

$$
\alpha(f)=\int_{\mathbb{R}} f(t) U_{t} d t
$$

defines an operator in $\mathbb{B}(E)$ and the map $\alpha$ can be extended to a nondegenerate ${ }^{*}$-homomorphism $\hat{\alpha}: C^{*}(\mathbb{R}) \rightarrow \mathbb{B}(E)$. Moreover, $\hat{\alpha}$ can be extended to a *-homomorphism $\check{\alpha}: \mathcal{M}\left(C^{*}(\mathbb{R})\right) \rightarrow \mathbb{B}(E)$ ([RW98, Proposition C.17]), where $\mathcal{M}\left(C^{*}(\mathbb{R})\right.$ ) denotes the algebra of double centralizers of the algebra $C^{*}(\mathbb{R})$.

Let $t \in \mathbb{R}$. Define

$$
\lambda_{t}: L^{1}(\mathbb{R}) \rightarrow L^{1}(\mathbb{R}), f \mapsto(s \mapsto f(s-t)) .
$$

Using the translation invariance of the Lebesgue measure, we get $\lambda_{t} \in \mathcal{M}\left(L^{1}(\mathbb{R})\right)$. By approximating any $f \in C^{*}(\mathbb{R})$ by elements of $L^{1}(\mathbb{R})$, we can define the double centralizer $\hat{\lambda}_{t} \in \mathcal{M}\left(C^{*}(\mathbb{R})\right)$ using $\lambda_{t}$. For all $f \in C_{c}(\mathbb{R})$ and $x \in E$ we obtain

$$
\check{\alpha}\left(\hat{\lambda}_{t}\right) \alpha(f) x=\alpha\left(\lambda_{t} f\right) x=U_{t} \alpha(f) x
$$

and thus

$$
\check{\alpha}\left(\hat{\lambda}_{t}\right)=U_{t} .
$$

The Fourier transform $\mathscr{F}: L^{1}(\mathbb{R}) \rightarrow C_{0}(\hat{\mathbb{R}}) \cong C_{0}(\mathbb{R})$ can be extended to a ${ }^{*}$-isomorphism $\hat{\mathscr{F}}: C^{*}(\mathbb{R}) \rightarrow C_{0}(\mathbb{R})\left(\left[\right.\right.$ RW98, Example C.20]). Denote by $C_{b}(\mathbb{R})$ the set of bounded continuous functions on $\mathbb{R}$. Because there is an embedding of $C_{0}(\mathbb{R})$ into $\mathcal{M}\left(C_{0}(\mathbb{R})\right) \cong C_{b}(\mathbb{R})$, we can regard $\hat{\mathscr{F}}$ as a map from $C^{*}(\mathbb{R})$ to $\mathcal{M}\left(C_{0}(\mathbb{R})\right)$. Using [Lan95, Proposition 2.5], we can extend this nondegenerate *-homomorphism to a *-homomorphism $\check{\mathscr{F}}: \mathcal{M}\left(C^{*}(\mathbb{R})\right) \rightarrow \mathcal{M}\left(C_{0}(\mathbb{R})\right)$ and conclude

$$
\check{\mathscr{F}}\left(\hat{\lambda}_{t}\right)=e_{t} \text {. }
$$

The nondegenerate ${ }^{*}$-homomorphism $\beta:=\hat{\alpha} \circ \hat{\mathscr{F}}^{-1}: C_{0}(\mathbb{R}) \rightarrow \mathbb{B}(E)$ extends to a *-homomorphism $\check{\beta}: \mathcal{M}\left(C_{0}(\mathbb{R})\right) \rightarrow \mathbb{B}(E)$. Recall that $\iota$ is the canonical inclusion of $\mathbb{R}$ into $\mathbb{C}$. Using $\iota \in C(\mathbb{R}) \cong \mathcal{R}\left(C_{0}(\mathbb{R})\right)$ ([Wor91, Example 2]) and [Lan95, Proposition 10.7], it follows that $A:=\beta(\iota)$ is a self-adjoint regular operator on $E$. Because $\varphi_{A}(\iota)=A=\beta(\iota)$ we conclude with [Kus97, Result 6.3]: $\left.\varphi_{A}\right|_{C_{0}(\mathbb{R})}=\beta$. With Lan95, Proposition 2.1] it follows that $\left.\varphi_{A}\right|_{C_{b}(\mathbb{R})}=\check{\beta}$ and $\check{\beta} \circ \check{\mathscr{F}}=\check{\alpha}$. Together with (2.3) und (2.2), we obtain

$$
\exp (\mathrm{i} t A)=\varphi_{A}\left(e_{t}\right)=\check{\beta}\left(e_{t}\right)=\check{\beta}\left(\check{\mathscr{F}}\left(\hat{\lambda}_{t}\right)\right)=\check{\alpha}\left(\hat{\lambda}_{t}\right)=U_{t} .
$$

To prove (ii) we notice that there exists a unique nondegenerate injective *-

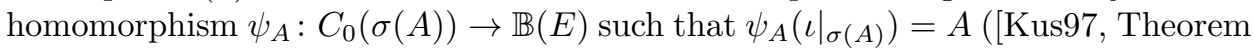
3.4]). Due to [Lan95, Proposition 2.5] the map $\psi_{A}$ can be extended to an injective ${ }^{*}$-homomorphism $\overline{\psi_{A}}: C_{b}(\sigma(A)) \rightarrow \mathbb{B}(E)$. With Kus97, Lemma 3.6] we have 
$\varphi_{A}(f)=\overline{\psi_{A}}\left(\left.f\right|_{\sigma(A)}\right)$ for all $f \in C(\mathbb{R})$. Denote by 1 the function that is constant 1 on $\mathbb{R}$. We conclude that

$$
\left\|U_{t}-\mathrm{id}_{E}\right\|=\left\|\varphi_{A}\left(e_{t}-\mathbf{1}\right)\right\|=\left\|\overline{\psi_{A}}\left(\left.\left(e_{t}-\mathbf{1}\right)\right|_{\sigma(A)}\right)\right\|=\left\|\left.\left(e_{t}-\mathbf{1}\right)\right|_{\sigma(A)}\right\| .
$$

Because $\left(U_{t}\right)_{t \in \mathbb{R}}$ is norm-continuous, $\sigma(A)$ is bounded. Therefore $\left.\iota\right|_{\sigma(A)}$ is bounded and we get: $A=\psi_{A}\left(\left.\iota\right|_{\sigma(A)}\right) \in \mathbb{B}(E)$.

2.3. Operator spaces. This section discusses basic results from operator space theory that we will need later in the paper (see e.g. [ER00, Pau02, Pis03] or BLM04).

Let $H$ be a Hilbert space. Denote by $L(H)$ the algebra of linear, continuous operators on $H$. A (concrete) operator space is a closed subspace $X$ of $L(H)$. Let $M_{m, n}(X)$ denote the space of $m \times n$ matrices with entries in $X$, and set $M_{n}(X):=$ $M_{n, n}(X)$. We may view the space $M_{n}(X)$ as a subspace of $L\left(H^{n}\right)$. Thus this matrix space has a natural norm $\|\cdot\|_{n}$ for each $n \in \mathbb{N}$. Note that operator spaces can be characterized intrinsically.

Let $X, Y$ be operator spaces and $\alpha: X \rightarrow Y$ be linear. Define

$$
\alpha_{n}:=\alpha^{(n)}: M_{n}(X) \rightarrow M_{n}(Y), x \mapsto\left(\alpha\left(x_{i j}\right)\right)_{i, j},
$$

called the $n$th amplification of $\alpha$. The map $\alpha$ is called completely contractive (resp. completely isometric) if $\alpha_{n}$ is contractive (resp. isometric) for all $n \in \mathbb{N}$. Furthermore, $\alpha$ is called completely bounded if $\|\alpha\|_{\text {cb }}:=\sup _{n \in \mathbb{N}}\left\|\alpha_{n}\right\|<\infty$.

An operator space $X$ is called unital if it has a distinguished element $e_{X}$ such that there exist a $C^{*}$-algebra $\mathfrak{A}$ with unit $e_{\mathfrak{A}}$ and a completely isometric map $\eta: X \rightarrow \mathfrak{A}$ satisfying $\eta\left(e_{X}\right)=e_{\mathfrak{A}}$. Observe that unital operator spaces can be characterized intrinsically ([BN11], $\mathrm{HN} 12])$.

Recall that an operator space $X$ is called injective if for every operator space $Y \subseteq Z$ and every completely contractive map $\varphi: Y \rightarrow X$ there is a completely contractive map $\Phi: Z \rightarrow X$ such that $\left.\Phi\right|_{Y}=\varphi$. For every operator space $X$ there exists a unique injective envelope $\left(I(X), j_{X}\right)$, i. e., a completely isometric map $j_{X}: X \rightarrow I(X)$ and an injective operator space $I(X)$ such that for every injective subspace $Y$ of $I(X)$ satisfying $j_{X}(X) \subseteq Y$ we have $Y=I(X)$.

Let $X \subseteq L(H)$ be an operator space. We can embed $X$ into an operator system, i. e., a self-adjoint closed subspace of $L(H)$ containing $\mathrm{id}_{H}$. To accomplish this we form the Paulsen system

$$
\mathcal{S}(X):=\left(\begin{array}{cc}
\mathbb{C i d}_{H} & X \\
X^{*} & \mathbb{C i d}_{H}
\end{array}\right) \subseteq M_{2}(L(H)) .
$$

There exists a completely contractive map $\Phi: M_{2}(L(H)) \rightarrow M_{2}(L(H))$ such that the image of $\Phi$ is an injective envelope of the operator space $\mathcal{S}(X)$. Moreover, $I(\mathcal{S}(X))$ is a unital $C^{*}$-algebra with multiplication $x \cdot y:=\Phi(x y)$. Let $p_{1}:=\operatorname{id}_{H} \oplus 0$ and $p_{2}:=0 \oplus \mathrm{id}_{H}$ be the canonical projections in $I(\mathcal{S}(X))$. Then $p_{1}$ and $p_{2}$ are orthogonal and corner-preserving. Therefore, with respect to $p_{1}$ and $p_{2}$, we may decompose $I(\mathcal{S}(X))$ as

$$
I(\mathcal{S}(X))=\left(\begin{array}{cc}
I_{11}(X) & I_{12}(X) \\
I_{12}(X)^{*} & I_{22}(X)
\end{array}\right)
$$

and have $I_{12}(X)=I(X)$. 
The following operator spaces will play a crucial role in this article.

If $\mathfrak{A}$ is a $C^{*}$-algebra, then the ${ }^{*}$-algebra $M_{n}(\mathfrak{A})$ has a unique norm such that $M_{n}(\mathfrak{A})$ is a $C^{*}$-algebra. With respect to these norms, $\mathfrak{A}$ is an operator space.

Let $X$ be an operator space and $m, n \in \mathbb{N}$. We can regard $M_{m, n}(X)$ as a subspace of $M_{p}(X)$, where $p:=\max \{m, n\}$. Thus $M_{m, n}(X)$ is an operator space. The set $C_{n}(X):=M_{n, 1}(X)$ is called a column operator space.

Furthermore, every Hilbert $C^{*}$-module $E$ over a $C^{*}$-algebra $\mathfrak{A}$ carries the structure of an operator space. For every $n \in \mathbb{N}$ and $x \in M_{n}(E)$,

$$
\|x\|_{n}=\left\|\left(\sum_{k=1}^{n}\left\langle x_{k i}, x_{k j}\right\rangle\right)_{i, j}\right\|^{1 / 2}
$$

defines a norm such that $E$ becomes an operator space.

2.4. Multipliers on operator spaces. Let us recall some facts about multipliers on operator spaces (see e.g. [BLM04]). These multipliers generalize multipliers on $C^{*}$-algebras.

Definition 2.7. Let $X$ be an operator space and $T: X \rightarrow X$ be linear.

(i) $T$ is called a (left) multiplier on $X$ if there exist a Hilbert space $H$ together with a completely isometric map $\eta: X \rightarrow L(H)$ and $a \in L(H)$ such that $\eta(T x)=a \eta(x)$ for all $x \in X$. The set of all multipliers on $X$ is denoted by $\mathscr{M}_{l}(X)$.

(ii) For all $T \in \mathscr{M}_{l}(X)$ define the multiplier norm $\|T\|_{\mathscr{M}_{l}(X)}:=\inf \{\|a\| \mid$ there exist a Hilbert space $H$, a completely isometric map $\eta: X \rightarrow L(H)$ and $a \in L(H)$ s.t. $\forall x \in X: \eta(T x)=a \eta(x)\}$.

(iii) $T$ is called a left adjointable multiplier on $X$ if there exist a Hilbert space $H$, a completely isometric map $\eta: X \rightarrow L(H)$ and a map $S: X \rightarrow X$ such that $\eta(T x)^{*} \eta(y)=\eta(x)^{*} \eta(S y)$ for all $x, y \in X$. The set of all left adjointable multipliers on $X$ is denoted by $\mathscr{A}_{l}(X)$.

For any operator space $X$ we have $\mathscr{M}_{l}(X) \subseteq \mathscr{A}_{l}(X)$. Moreover, $\mathscr{A}_{l}(X)$ is a unital $C^{*}$-algebra. The multiplier algebra of a $C^{*}$-algebra $\mathfrak{A}$ is isomorphic to $\mathscr{A}_{l}(\mathfrak{A})$. If $E$ is a Hilbert $C^{*}$-module, then

$$
\mathscr{A}_{l}(E)=\mathbb{B}(E)
$$

([BLM04, Corollary 8.4.2]). Define

$$
\begin{aligned}
& \mathcal{I} M_{\ell}(X):=\left\{a \in I_{11}(X) \mid a \cdot X \subseteq X\right\} \text { and } \\
& \mathcal{I} M_{\ell}^{*}(X):=\mathcal{I} M_{\ell}(X) \cap \mathcal{I} M_{\ell}(X)^{*} .
\end{aligned}
$$

Then $\mathcal{I} M_{\ell}(X)\left(\right.$ resp. $\left.\mathcal{I} M_{\ell}^{*}(X)\right)$ is isometrically isomorphic to $\mathscr{M}_{l}(X)\left(\right.$ resp. $\left.\mathscr{A}_{l}(X)\right)$.

Left multipliers are characterized intrinsically by the following result:

Theorem 2.8 ([BEZ02, Theorem 1.1], Wer04, Corollary 3.13]). Let $X$ be an operator space, $T: X \rightarrow X$ be linear and $\lambda>0$. The following assertions are equivalent:

(a) $T \in \mathscr{M}_{l}(X)$ satisfies $\|T\|_{\mathscr{M}_{l}(X)} \leq \frac{1}{\lambda}$. 
(b) The map

$$
\tau_{\lambda T}: C_{2}(X) \rightarrow C_{2}(X),\left(\begin{array}{c}
x \\
y
\end{array}\right) \mapsto\left(\begin{array}{c}
\lambda T x \\
y
\end{array}\right),
$$

is completely contractive.

Proposition 2.9 (cf. [Wer99, Corollary 4.3], Ble01, p. 308]). Let X be an operator space and $U: X \rightarrow X$ be linear. The following assertions are equivalent:

(a) $U$ is a unitary element of $\mathscr{A}_{l}(X)$.

(b) The map

$$
\tau_{U}: C_{2}(X) \rightarrow C_{2}(X),\left(\begin{array}{l}
x \\
y
\end{array}\right) \mapsto\left(\begin{array}{c}
U x \\
y
\end{array}\right),
$$

is a completely isometric isomorphism.

(c) There exists a unitary $u \in \mathcal{I} M_{\ell}^{*}(X)$ such that $j(U x)=u \cdot j(x)$ for all $x \in X$.

\section{UNBOUNDED MULTIPLIERS ON OPERATOR SPACES}

In this section we will introduce three different definitions of unbounded multipliers on an operator space.

3.1. Unbounded skew-adjoint multipliers. An operator space has less structure than a Hilbert $C^{*}$-module $E$. Therefore it is not obvious how to define an unbounded multiplier on an operator space. We know that the set $\mathbb{B}(E)$ of adjointable operators is isomorphic to the set $\mathscr{A}_{l}(E)$, where $E$ is equipped with the canonical operator space structure of equation (2.4). The fact that skew-adjoint regular operators can be characterized using $C_{0}$-groups (Theorems 2.6] and 2.5) motivates the following definition:

Definition 3.1. Let $X$ be an operator space.

(i) An operator $A: D(A) \subseteq X \rightarrow X$ is called a $C_{0}$-left multiplier if $A$ generates a $C_{0}$-semigroup $\left(T_{t}\right)_{t \geq 0}$ on $X$ satisfying $T_{t} \in \mathscr{M}_{l}(X)$ for all $t \geq 0$.

(ii) An operator $A: D(A) \subseteq X \rightarrow X$ is called an unbounded skew-adjoint multiplier if $A$ generates a $C_{0}$-group $\left(U_{t}\right)_{t \in \mathbb{R}}$ on $X$ satisfying $U_{t} \in \mathscr{A}_{l}(X)$ for all $t \in \mathbb{R}$.

(iii) The set of all $C_{0}$-left multipliers (resp. unbounded skew-adjoint multipliers) on $X$ is denoted by $\mathscr{M}_{l}^{C_{0}}(X)$ (resp. $\left.\mathscr{A}_{l, s}^{C_{0}}(X)\right)$.

Using the fact that $t \mapsto \mathrm{e}^{t A}(A \in L(X))$ defines a norm-continuous semigroup with generator $A$ and [EN00, Proposition I.3.5], we have the following, similar to equation (2.1):

Proposition 3.2. Let $X$ be an operator space. Then:

(i) $\mathscr{A}_{l, s}^{C_{0}}(X) \subseteq \mathscr{M}_{l}^{C_{0}}(X)$.

(ii) $\mathscr{M}_{l}(X)=\left\{A \in \mathscr{M}_{l}^{C_{0}}(X) \mid D(A)=X\right\}$.

(iii) $\left\{A \in \mathscr{A}_{l}(X) \mid A\right.$ skew-adjoint $\}=\left\{A \in \mathscr{A}_{l, s}^{C_{0}}(X) \mid D(A)=X\right\}$.

In a unital $C^{*}$-algebra $\mathfrak{A}$ the set of (bounded) multipliers is equal to $\mathcal{R}(\mathfrak{A})$. A similar result holds for operator spaces:

Proposition 3.3. If $X$ is a unital operator space, we have

(i) $\mathscr{M}_{l}(X)=\mathscr{M}_{l}^{C_{0}}(X)$ and

(ii) $\left\{A \in \mathscr{A}_{l}(X) \mid A\right.$ skew-adjoint $\}=\mathscr{A}_{l, s}^{C_{0}}(X)$. 
Proof. We will only prove (i), because the proof of (ii) is similar to the proof of (i). Let $A \in \mathscr{M}_{l}^{C_{0}}(X)$. Due to Proposition 3.2 it suffices to show $D(A)=X$.

Let $\left(T_{t}\right)_{t \geq 0}$ be the $C_{0}$-semigroup generated by $A$. For every $t \geq 0$ there exists an $a_{t} \in \mathcal{I} M_{\ell}(X)$ such that $j_{X}\left(T_{t} x\right)=a_{t} \cdot j_{X}(x)$ for all $x \in X$. There exists a Hilbert space $H$ such that $X \subseteq L(H)$ and $e_{X}=\operatorname{id}_{H}$. Because $X$ is unital, we have that $\|S\|_{\mathrm{cb}}=\|S\|_{\mathscr{M}_{l}(X)}$ for all $S \in \mathscr{M}_{l}(X)$ ([Ble01, p. 20]) and that the embedding $j_{X}: X \rightarrow I(X)$ is unital ([BLM04, Corollary 4.2.8]). Together we obtain that

$$
\begin{aligned}
\left\|T_{t}-\mathrm{id}_{X}\right\| & \leq\left\|T_{t}-\mathrm{id}_{X}\right\|_{\mathrm{cb}}=\left\|T_{t}-\mathrm{id}_{X}\right\|_{\mathscr{M}_{l}(X)}=\left\|a_{t}-e_{I_{11}(X)}\right\| \\
& =\left\|a_{t} \cdot e_{I(X)}-e_{I(X)}\right\|=\left\|a_{t} \cdot j_{X}\left(e_{X}\right)-j_{X}\left(e_{X}\right)\right\|=\left\|T_{t}\left(e_{X}\right)-e_{X}\right\|
\end{aligned}
$$

converges to 0 for $t \rightarrow 0$. Thus $\left(T_{t}\right)_{t \geq 0}$ is norm-continuous. We conclude that $D(A)=X$, and the assertion follows with Proposition 3.2

Theorem 3.4. For a Hilbert $C^{*}$-module $E$, we have

$$
\{A \in \mathcal{R}(E) \mid A \text { skew-adjoint }\}=\mathscr{A}_{l, s}^{C_{0}}(E) .
$$

Proof. Let $A \in \mathcal{R}(E)$ be skew-adjoint. Then $h:=-\mathrm{i} A$ is self-adjoint. Moreover $U_{t}:=\exp (\mathrm{ith})$ defines a $C_{0}$-group on $E$ with $U_{t} \in \mathbb{B}(E)=\mathscr{A}_{l}(E)$ unitary and with generator i $h=A$ due to Theorem 2.5] and equation (2.5); thus $A \in \mathscr{A}_{l, s}^{C_{0}}(E)$.

The other inclusion follows with Theorem 2.6. Theorem 2.5 and the uniqueness of the generator of a $C_{0}$-group.

3.2. Unbounded multipliers. In this section we will generalize the definition of regular operators on Hilbert $C^{*}$-modules to operator spaces.

Let $A_{i}: D\left(A_{i}\right) \subseteq X \rightarrow X$ be an operator for all $i \in\{1, \ldots, 4\}$. Define

$$
\begin{gathered}
\left(\begin{array}{cc}
A_{1} & A_{2} \\
A_{3} & A_{4}
\end{array}\right): \quad\left(D\left(A_{1}\right) \cap D\left(A_{3}\right)\right) \times\left(D\left(A_{2}\right) \cap D\left(A_{4}\right)\right) \subseteq C_{2}(X) \rightarrow C_{2}(X), \\
(x, y) \mapsto\left(A_{1} x+A_{2} y, A_{3} x+A_{4} y\right) .
\end{gathered}
$$

To introduce the notion of an unbounded multiplier we use the fact that i $\left(\begin{array}{cc}0 & A \\ A^{*} & 0\end{array}\right)$ is a skew-adjoint regular operator on $E \oplus E$ for every $A \in \mathcal{R}(E)$. This motivates the following definition:

Definition 3.5. Let $X$ be an operator space. A map $A: D(A) \subseteq X \rightarrow X$ is called an unbounded multiplier if there exists $B: D(B) \subseteq X \rightarrow X$ satisfying i $\left(\begin{array}{cc}0 & A \\ B & 0\end{array}\right) \in$ $\mathscr{A}_{l, s}^{C_{0}}\left(C_{2}(X)\right)$. The set of all unbounded multipliers on $X$ is denoted by $\mathscr{A}_{l}^{C_{0}}(X)$.

Every unbounded multiplier is densely defined and closed. We will show in Theorem 3.20 that $B$ is unique. If $X$ is a Hilbert $C^{*}$-module, we will obtain $B=A^{*}$ (Theorem 3.8). Therefore $B$ can be viewed as the adjoint of $A$.

Proposition 3.6. Let $X$ be an operator space. Then:

(i) $\mathscr{A}_{l}(X) \subseteq \mathscr{A}_{l}^{C_{0}}(X)$.

(ii) If $X$ is unital, we have $\mathscr{A}_{l}(X)=\mathscr{A}_{l}^{C_{0}}(X)$.

Proof. To prove (i) let $A \in \mathscr{A}_{l}(X)$. Then $\tilde{A}:=\mathrm{i}\left(\begin{array}{cc}0 & A \\ A^{*} & 0\end{array}\right) \in \mathscr{A}_{l}\left(C_{2}(X)\right)$ is skewadjoint, where $A^{*}$ denotes the adjoint of $A$ in the $C^{*}$-algebra $\mathscr{A}_{l}(X)$. With Proposition 3.2 we conclude that $\tilde{A} \in \mathscr{A}_{l, s}^{C_{0}}\left(C_{2}(X)\right)$; thus $A \in \mathscr{A}_{l}^{C_{0}}(X)$.

Part (ii) is deduced by using Proposition 3.3 and the following lemma. 
The following is straightforward using [BP01, Corollary 1.3]:

Lemma 3.7 ([Sch09, Lemma 3.20]). Let $X$ be an operator space. Let $A: D(A) \subseteq$ $X \rightarrow X$ and $B: D(B) \subseteq X \rightarrow X$ be operators satisfying $\hat{A}:=\left(\begin{array}{cc}0 & A \\ B & 0\end{array}\right) \in \mathscr{A}_{l}\left(C_{2}(X)\right)$ and $(\hat{A})^{*}=\hat{A}$. Then $A, B \in \mathscr{A}_{l}(X)$ and $B^{*}=A$.

The following important theorem shows that on a Hilbert $C^{*}$-module unbounded multipliers coincide with regular operators:

Theorem 3.8. Let $E$ be a Hilbert $C^{*}$-module.

(i) $\mathscr{A}_{l}^{C_{0}}(E)=\mathcal{R}(E)$.

(ii) Let $A \in \mathscr{A}_{l}^{C_{0}}(E)$, and suppose there exists $B: D(B) \subseteq E \rightarrow E$ such that $\mathrm{i}\left(\begin{array}{cc}0 & A \\ B & 0\end{array}\right) \in \mathscr{A}_{l, s}^{C_{0}}\left(C_{2}(E)\right)$. Then $A^{*}=B \in \mathcal{R}(E)$.

Proof. We first show (ii) and the inclusion ' $\subseteq$ ' of (i). Let $A \in \mathscr{A}_{l}^{C_{0}}(E)$ and $\hat{A}:=$ $\left(\begin{array}{cc}0 & A \\ B & 0\end{array}\right)$. The direct sum $E \oplus E$ is equipped with the inner product

$$
\left\langle\left(x_{1}, y_{1}\right),\left(x_{2}, y_{2}\right)\right\rangle_{E \oplus E}:=\left\langle x_{1}, x_{2}\right\rangle_{E}+\left\langle y_{1}, y_{2}\right\rangle_{E},
$$

a Hilbert $C^{*}$-module. Moreover $E \oplus E$ is isometrically isomorphic to the column operator space $C_{2}(E)$ of $E$ viewed as an operator space (Sch09, Proposition 2.14]).

Both $A$ and $B$ are densely defined and closed. Using Theorem 3.4 we obtain

$$
\mathrm{i} \hat{A} \in \mathscr{A}_{l, s}^{C_{0}}\left(C_{2}(E)\right) \cong\left\{R \in \mathcal{R}(E \oplus E) \mid R^{*}=-R\right\} ;
$$

thus $\left(\begin{array}{cc}0 & B^{*} \\ A^{*} & 0\end{array}\right)=(\hat{A})^{*}=\hat{A}=\left(\begin{array}{cc}0 & A \\ B & 0\end{array}\right)$. We conclude: $B=A^{*}$. Therefore $A^{*}$ is densely defined. A simple calculation shows that $1+A^{*} A$ has dense image. It follows that $A$ is regular. With Lan95, Corollary 9.6] we get $A^{*} \in \mathcal{R}(E)$.

To prove the inclusion ' $\supseteq$ ' of (i) let $A \in \mathcal{R}(E)$. Then $\hat{A}:=\left(\begin{array}{cc}0 & A \\ A^{*} & 0\end{array}\right)$ is regular and self-adjoint. With equation (3.1) we obtain i $\hat{A} \in \mathscr{A}_{l, s}^{C_{0}}\left(C_{2}(E)\right)$; thus $A \in$ $\mathscr{A}_{l}^{C_{0}}(E)$.

3.3. Characterizations of unbounded multipliers. Our next goal is to characterize unbounded multipliers. Let us recall the following two definitions:

Definition 3.9. Let $H, K$ be Hilbert spaces. A ternary ring of operators (TRO) is a closed subspace $Z$ of $L(H, K)$ such that $Z Z^{*} Z \subseteq Z$. A subtriple of a TRO $Z$ is a closed subspace $Y$ of $Z$ satisfying $Y Y^{*} Y \subseteq Y$.

Every TRO $Z$ is a full Hilbert $C^{*}$-module over the $C^{*}$-algebra $Z^{*} Z$. Conversely, every Hilbert $C^{*}$-module can be represented faithfully as a TRO. We can view the injective envelope $I(X)$ as a TRO.

Definition 3.10. The triple envelope $\mathcal{T}(X)$ of an operator space $X$ is the smallest subtriple of $I(X)$ containing $X$.

We have

$$
\mathcal{T}(X)=\varlimsup\left\{x_{1} \cdot x_{2}^{*} \cdot x_{3} \cdot x_{4}^{*} \cdots x_{2 n+1} \mid n \geq 0, x_{1}, \ldots, x_{2 n+1} \in j(X)\right\} .
$$

The following is straightforward from Har81, p. 341]:

Lemma 3.11. Let $X$ be an operator space. There exist a Hilbert space $K$, a unital injective ${ }^{*}$-representation $\pi: I(\mathcal{S}(X)) \rightarrow L(K)$ and closed subspaces $K_{1}, K_{2}$ of $K$ such that

(i) $\left[\pi(\mathcal{T}(X)) K_{1}\right]:=\operatorname{lin}\left\{x y \mid x \in \pi(\mathcal{T}(X)), y \in K_{1}\right\}$ is dense in $K_{2}$, 
(ii) $\left[\pi(\mathcal{T}(X)) K_{1}^{\perp}\right]=\{0\}$ and

(iii) $[\pi(j(X)) K] \subseteq K_{2}$.

Definition 3.12. The quadruple $\left(\pi, K, K_{1}, K_{2}\right)$, introduced in Lemma 3.11, is called a $\mathcal{T}(X)$-embedding of $X$.

In the following theorem it is shown that an unbounded multiplier can be characterized intrinsically (part (b)) or with the help of a $C_{0}$-group on a Hilbert space (part $(\mathrm{d})$ ) and that an unbounded multiplier is essentially the restriction of a regular operator on the Hilbert $C^{*}$-module $\mathcal{T}(X)$ (part (c)).

Theorem 3.13. Let $X$ be an operator space. Let $\left(\pi, K, K_{1}, K_{2}\right)$ be a $\mathcal{T}\left(C_{2}(X)\right)$ embedding of $C_{2}(X)$, and set $\eta:=\pi \circ j_{C_{2}(X)}: C_{2}(X) \rightarrow L(K)$. Let $A: D(A) \subseteq$ $X \rightarrow X$ be linear. The following assertions are equivalent:

(a) $A \in \mathscr{A}_{l}^{C_{0}}(X)$.

(b) There exists a map $B: D(B) \subseteq X \rightarrow X$ such that $\mathrm{i}\left(\begin{array}{cc}0 & A \\ B & 0\end{array}\right) \oplus\left(\begin{array}{ll}0 & 0 \\ 0 & 0\end{array}\right)$ generates a completely contractive $C_{0}$-group on $C_{2}\left(C_{2}(X)\right) \cong C_{4}(X)$.

(c) There exists $B \in \mathcal{R}(\mathcal{T}(X))$ with the following properties:

(i) $j \circ A=\left.B \circ j\right|_{D(A)}$ and

(ii) there exist $\lambda, \mu>0$ such that the restriction of

$$
\begin{array}{r}
\lambda-\mathrm{i}\left(\begin{array}{cc}
0 & B \\
B^{*} & 0
\end{array}\right) \quad \text { resp. } \quad \mu+\mathrm{i}\left(\begin{array}{cc}
0 & B \\
B^{*} & 0
\end{array}\right) \\
\text { to } j_{C_{2}(X)}\left(C_{2}(X)\right) \text { is surjective onto } j_{C_{2}(X)}\left(C_{2}(X)\right) .
\end{array}
$$

(d) There exist a map $B: D(B) \subseteq X \rightarrow X$ such that $\tilde{A}:=\mathrm{i}\left(\begin{array}{cc}0 & A \\ B & 0\end{array}\right)$ is densely defined with $\rho(\tilde{A}) \neq \emptyset$ and a $C_{0}$-group $\left(b_{t}\right)_{t \in \mathbb{R}}$ consisting of unitary elements of $K_{2}$ such that:

(i) $L_{b_{t}}: \eta\left(C_{2}(X)\right) \rightarrow \eta\left(C_{2}(X)\right), y \mapsto b_{t} y$, and $L_{b_{t}^{*}}$ define $C_{0}$-groups on the space $\eta\left(C_{2}(X)\right)$ and

(ii) for the generator $C$ of $\left(L_{b_{t}}\right)_{t \in \mathbb{R}}$, we have $\eta(D(\tilde{A})) \subseteq D(C)$.

Note that part (c)(i) of the above theorem implies that $j(D(A)) \subseteq D(B)$.

A first step in the proof of the above characterization is the following result:

Theorem 3.14. Let $X$ be an operator space and $A: D(A) \subseteq X \rightarrow X$. Then $A \in \mathscr{A}_{l, s}^{C_{0}}(X)$ if and only if $A \oplus 0$ generates a completely contractive $C_{0}$-group on $C_{2}(X)$.

Proof. Let $A \in \mathscr{A}_{l, s}^{C_{0}}(X)$ and denote by $\left(T_{t}\right)_{t \in \mathbb{R}}$ the $C_{0}$-group generated by $A$. Due to Proposition 2.9], $\left(T_{t} \oplus \mathrm{id}_{X}\right)_{t \in \mathbb{R}}$ is a completely contractive $C_{0}$-group, which is clearly generated by $A \oplus 0$.

Conversely, let $\left(S_{t}\right)_{t \in \mathbb{R}}$ be the completely contractive $C_{0}$-group generated by $A \oplus 0$. We conclude that $S_{t}=T_{t} \oplus \mathrm{id}_{X}$, where $\left(T_{t}\right)_{t \in \mathbb{R}}$ is the $C_{0}$-group generated by $A$. Because $S_{t}$ is a completely isometric isomorphism for all $t \in \mathbb{R}$ the assertion follows with Proposition 2.9 .

With this result we conclude that (a) and (b) in Theorem 3.13 are equivalent. Thus unbounded multipliers can be characterized using generators of completely contractive $C_{0}$-groups. Such generators are analyzed in the following two theorems, which generalize well-known results from the theory of contractive $C_{0}$-semigroups to the setting of completely contractive (c. c.) $C_{0}$-semigroups on an operator space $X$. By using the classical result on each matrix level, one can prove the following theorem. For details see [Sch09, Satz 3.33]. 
Theorem 3.15 (Theorem of Hille-Yosida for c.c. $C_{0}$-semigroups). Let $X$ be an operator space and $A: D(A) \subseteq X \rightarrow X$ be linear. The following assertions are equivalent:

(a) $A$ is the generator of a completely contractive $C_{0}$-semigroup on $X$.

(b) $A$ is densely defined and closed, $\mathbb{R}_{>0}$ is a subset of the resolvent set $\rho(A)$ of $A$ and

$$
\left\|\lambda(\lambda-A)^{-1}\right\|_{c b} \leq 1 \quad \text { for all } \lambda>0 .
$$

An operator $A: D(A) \subseteq X \rightarrow X$ is called completely dissipative if $A_{n}$ is dissipative for all $n \in \mathbb{N}$, i. e.

$$
\left\|\left(\lambda-A_{n}\right) x\right\| \geq \lambda\|x\| \quad \text { for all } \lambda>0 \text { and } x \in D\left(A_{n}\right) .
$$

Theorem 3.16 (Theorem of Lumer-Phillips for c.c. $C_{0}$-semigroups). Let $X$ be an operator space and $A: D(A) \subseteq X \rightarrow X$ be linear and densely defined. Then $A$ generates a completely contractive $C_{0}$-semigroup if and only if $A$ is completely dissipative and there exists a $\lambda>0$ such that $\lambda-A$ is surjective onto $X$.

Proof. The direction ' $\Rightarrow$ ' is a consequence of Theorem 3.15, because

$$
\left\|(\lambda-A)_{n} x\right\| \geq \frac{1}{\left\|(\lambda-A)_{n}^{-1} x\right\|} \geq \lambda\|x\|
$$

for all $\lambda>0, n \in \mathbb{N}$ and $x \in D\left(A_{n}\right)$.

The other direction follows from the theorem of Lumer-Phillips for contractive $C_{0}$-semigroups ([Wer07, Theorem VII.4.16]).

Using the two theorems above together with the equivalence of (a) and (b) in Theorem 3.13, we obtain the following theorem. For details see [Sch09, Proposition 3.41].

Theorem 3.17. Let $X$ be an operator space and $A: D(A) \subseteq X \rightarrow X$ be linear. The following assertions are equivalent:

(a) $A \in \mathscr{A}_{l}^{C_{0}}(X)$.

(b) There exists $B: D(B) \subseteq X \rightarrow X$ such that $\tilde{A}:=\mathrm{i}\left(\begin{array}{cc}0 & A \\ B & 0\end{array}\right)$ is densely defined and closed satisfying $\mathbb{R} \backslash\{0\} \subseteq \rho(\tilde{A})$ and

$$
\left\||\lambda|(\lambda-(\tilde{A} \oplus 0))^{-1}\right\|_{c b} \leq 1 \quad \text { for all } \lambda \in \mathbb{R} \backslash\{0\},
$$

where $\tilde{A} \oplus 0=\tilde{A} \oplus\left(\begin{array}{ll}0 & 0 \\ 0 & 0\end{array}\right)$ is an operator on $C_{2}\left(C_{2}(X)\right) \cong C_{4}(X)$.

(c) There exists $B: D(B) \subseteq X \rightarrow X$ with the following properties:

(i) $\tilde{A}:=\mathrm{i}\left(\begin{array}{cc}0 & A \\ B & 0\end{array}\right)$ is densely defined,

(ii) $\tilde{A} \oplus 0$ and $-\tilde{A} \oplus 0$ are completely dissipative and

(iii) there exist $\lambda, \mu>0$ such that $\lambda-\tilde{A}$ and $\mu+\tilde{A}$ are surjective onto $C_{2}(X)$.

The following result shows that we can lift unbounded multipliers from an operator space $X$ to the ternary envelope $\mathcal{T}(X)$ :

Proposition 3.18. Let $X$ be an operator space and $A \in \mathscr{A}_{l, s}^{C_{0}}(X)$ (resp. $A \in$ $\mathscr{A}_{l}^{C_{0}}(X)$ ). There exists a unique $B \in \mathscr{A}_{l, s}^{C_{0}}(\mathcal{T}(X))$ (resp. $B \in \mathscr{A}_{l}^{C_{0}}(\mathcal{T}(X))$ ) such that $j \circ A=\left.B \circ j\right|_{D(A)}$. 
Proof. The case $A \in \mathscr{A}_{l}^{C_{0}}(X)$ follows directly from the assertion for $\mathscr{A}_{l, s}^{C_{0}}(X)$.

Therefore let $A \in \mathscr{A}_{l, s}^{C_{0}}(X)$. Let $\left(T_{t}\right)_{t \in \mathbb{R}}$ be the $C_{0}$-group generated by $A$. There exists $a_{t} \in \mathcal{I} M_{\ell}^{*}(X)$ unitary such that $j\left(T_{t} x\right)=a_{t} \cdot j(x)$ for all $x \in X$. Define $S_{t}: \mathcal{T}(X) \rightarrow \mathcal{T}(X), z \mapsto a_{t} \cdot z$. Then $S_{t}$ is a unitary element of $\mathscr{A}_{l}(X)$ for all $t \in \mathbb{R}$. We conclude that

$$
\left\|a_{t} \cdot\left(j(x) \cdot j(y)^{*} \cdot j(z)\right)-j(x) \cdot j(y)^{*} \cdot j(z)\right\| \leq\left\|T_{t} x-x\right\|\left\|j(y)^{*} \cdot j(z)\right\| \rightarrow 0
$$

for $t \rightarrow 0$ and for all $x, y, z \in X$. Equation (3.2) implies that the set

$$
V:=\operatorname{lin}\left\{x_{1} \cdot x_{2}^{*} \cdot x_{3} \cdot x_{4}^{*} \cdots x_{2 n+1} \mid n \geq 0, x_{1}, \ldots, x_{2 n+1} \in j(X)\right\}
$$

is dense in $\mathcal{T}(X)$. It follows that $\lim _{t \rightarrow 0} S_{t} x=\lim _{t \rightarrow 0} a_{t} \cdot z=z$ for all $z \in V$ and $I_{11}(\mathcal{T}(X)) \cong I_{11}(X)$ ([Sch09, Proposition A.6]). We obtain

$$
\left\|S_{t} z\right\|_{\mathcal{T}(X)}=\left\|a_{t} \cdot z\right\|_{\mathcal{T}(X)} \leq\left\|a_{t}\right\|_{I_{11}(\mathcal{T}(X))}\|z\|_{\mathcal{T}(X)}=\left\|a_{t}\right\|_{I_{11}(X)}\|z\|_{\mathcal{T}(X)}
$$

for all $t \in \mathbb{R}$ and $z \in \mathcal{T}(X)$; thus $\left\|S_{t}\right\| \leq\left\|a_{t}\right\|=\left\|T_{t}\right\|_{\mathscr{M}_{l}(X)}=\left\|T_{t}\right\|$. With the characterization of $C_{0}$-semigroups ([EN00, Proposition I.5.3]) we conclude that $\left(S_{t}\right)_{t \in \mathbb{R}}$ is a $C_{0}$-group.

Let $B$ denote the generator of $\left(S_{t}\right)_{t \in \mathbb{R}}$. Then $B \in \mathscr{A}_{l, s}^{C_{0}}(\mathcal{T}(X))$ and

$$
j(A x)=\lim _{t \rightarrow 0} \frac{a_{t} \cdot j(x)-j(x)}{t}=B(j(x))
$$

for all $x \in D(A)$.

To show that $B$ is unique let $C \in \mathscr{A}_{l, s}^{C_{0}}(\mathcal{T}(X))$ such that $j \circ A=\left.C \circ j\right|_{D(A)}$. Define

$$
\begin{aligned}
& W:=W_{j(D(A)), j(X)}:=\operatorname{lin}\left\{x_{1} \cdot x_{2}^{*} \cdot x_{3} \cdot x_{4}^{*} \cdots x_{2 n+1} \mid\right. \\
&\left.n \geq 1, x_{1} \in j(D(A)), x_{2}, \ldots, x_{2 n+1} \in j(X)\right\} .
\end{aligned}
$$

With equation (3.4) we obtain $\left.B\right|_{W}=\left.C\right|_{W}$. Because $W$ is a core for $B$, i. e., $W$ is dense in $D(B)$ for the graph norm, it follows that $B=\overline{\left.B\right|_{W}}=\overline{\left.C\right|_{W}}=C$.

Proposition 3.19. Let $X, Y$ be operator spaces with $X \subseteq Y$ and $A \in \mathscr{A}_{l, s}^{C_{0}}(Y)$. Suppose there exist $\lambda, \mu>0$ such that $\lambda-\left.A\right|_{X}$ and $\mu+\left.A\right|_{X}$ are surjective onto $X$. Then $\left.A\right|_{X} \in \mathscr{A}_{l, s}^{C_{0}}(X)$.

Proof. Due to Theorem 3.14 we have that $A \oplus 0$ generates a completely contractive $C_{0}$-group on $C_{2}(Y)$. Define

$$
A_{\mid X}:=\{(x, y) \mid x \in D(A) \cap X, y=A x \in X\} .
$$

With the theorem of Lumer-Phillips (Theorem [3.16) $A \oplus 0$ and also $A_{\mid X} \oplus 0$ are completely dissipative and generate completely contractive $C_{0}$-groups. We conclude with Theorem 3.14; $A_{\mid X} \in \mathscr{A}_{l, s}^{C_{0}}(X)$. Let $\left(T_{t}\right)_{t \in \mathbb{R}}$ be the $C_{0}$-group generated by $A$. Because the $C_{0}$-group generated by $A_{\mid X}$ is equal to $\left(\left.T_{t}\right|_{X}\right)_{t \in \mathbb{R}}$, we obtain with [EN00, Corollary II.2.3]: $A_{\mid X}=\left.A\right|_{X}$.

Similar to regular operators, every unbounded multiplier has an adjoint:

Theorem 3.20. Let $X$ be an operator space and $A \in \mathscr{A}_{l}^{C_{0}}(X)$. There exists a unique operator $A^{*}: D\left(A^{*}\right) \subseteq X \rightarrow X$, called the adjoint of $A$, such that i $\left(\begin{array}{cc}0 & A \\ A^{*} & 0\end{array}\right) \in$ $\mathscr{A}_{l, s}^{C_{0}}\left(C_{2}(X)\right)$. We have $A^{*} \in \mathscr{A}_{l}^{C_{0}}(X)$ and $\left(A^{*}\right)^{*}=A$. 
Proof. The existence follows directly from the definition of an unbounded multiplier. To show the uniqueness, let $B_{i}: D\left(B_{i}\right) \subseteq X \rightarrow X(i \in\{1,2\})$ be such that $\tilde{B}_{i}:=\mathrm{i}\left(\begin{array}{cc}0 & A \\ B_{i} & 0\end{array}\right) \in \mathscr{A}_{l, s}^{C_{0}}\left(C_{2}(X)\right)$. Due to Proposition 3.18 , we find a regular operator $\tilde{C}_{i} \in \mathcal{R}\left(\mathcal{T}\left(C_{2}(X)\right)\right)$ satisfying $j \circ \tilde{B}_{i}=\left.\tilde{C}_{i} \circ j\right|_{D\left(\tilde{B}_{i}\right)}$. Let $\left(T_{t}^{(i)}\right)_{t \in \mathbb{R}}$ be the $C_{0}$-group generated by $\tilde{B}_{i}$. There exists $a_{t}^{(i)} \in \mathcal{I} M_{\ell}^{*}\left(C_{2}(X)\right)$ such that $j\left(T_{t}^{(i)} x\right)=a_{t}^{(i)} \cdot j(x)$ for all $x \in C_{2}(X)$. Note that $\tilde{C}_{i}$ is the generator of the $C_{0}$-group that is defined by $S_{t}^{(i)}: \mathcal{T}\left(C_{2}(X)\right) \rightarrow \mathcal{T}\left(C_{2}(X)\right), z \mapsto a_{t}^{(i)} \cdot z$. For all $k \in \mathbb{N} \cup\{0\}, x_{1} \in D\left(\tilde{B}_{i}\right)$ and $x_{2}, \ldots, x_{2 k+1} \in C_{2}(X)$ we obtain with $z:=j\left(x_{2}\right)^{*} \cdot j\left(x_{3}\right) \cdot j\left(x_{4}\right)^{*} \cdots j\left(x_{2 k+1}\right)$,

$$
j\left(\tilde{B}_{i}\left(x_{1}\right)\right) \cdot z=\lim _{t \rightarrow 0} \frac{a_{t}^{(i)} \cdot j\left(x_{1}\right)-j\left(x_{1}\right)}{t} \cdot z=\tilde{C}_{i}\left(j\left(x_{1}\right) \cdot z\right) .
$$

Set $W_{i}:=W_{D\left(\tilde{B}_{i}\right), C_{2}(X)}$. Using $\mathcal{T}\left(C_{2}(X)\right) \cong C_{2}(\mathcal{T}(X))$ ([BLM04, 8.3.12.(4)]), we conclude that $\left.\tilde{C}_{i}\right|_{W_{i}}$ has the same form as $\tilde{B}_{i}$, namely $\mathrm{i}\left(\begin{array}{c}0 * \\ *\end{array}\right)$. Moreover, $W_{i}$ is a core for $\tilde{C}_{i}$. Therefore, $\tilde{C}_{i}$ has the form i $\left(\begin{array}{cc}0 & * \\ * & 0\end{array}\right)$. Using Theorem 3.8 , there exists $\check{C}_{i} \in \mathcal{R}(\mathcal{T}(X))$ such that

$$
\tilde{C}_{i}=\mathrm{i}\left(\begin{array}{cc}
0 & \check{C}_{i} \\
\check{C}_{i}^{*} & 0
\end{array}\right)
$$

The set $W:=W_{j(D(A)), j(X)}$ is a core for $\check{C}_{1}$ and for $\check{C}_{2}$. Using $\left.\check{C}_{1}\right|_{W}=\left.\check{C}_{2}\right|_{W}$, we obtain

$$
\check{C}_{1}=\overline{\left.\check{C}_{1}\right|_{W}}=\overline{\left.\check{C}_{2}\right|_{W}}=\check{C}_{2} .
$$

It follows that $\tilde{C}_{1}=\tilde{C}_{2}$; thus $\tilde{B}_{1}=\tilde{B}_{2}$ and $B_{1}=B_{2}$.

With $\check{C}_{i}^{* *}=\check{C}_{i}\left(\right.$ Lan95, Corollary 9.4]) and $\check{C}_{i}^{*} \in \mathcal{R}(\mathcal{T}(X))$ (Lan95, Corollary 9.6]) we conclude that

$$
\hat{C}_{i}:=\mathrm{i}\left(\begin{array}{cc}
0 & \check{C}_{i}^{*} \\
\check{C}_{i} & 0
\end{array}\right) \in \mathcal{R}(\mathcal{T}(X) \oplus \mathcal{T}(X))
$$

is skew-adjoint. Using $\mathcal{T}(X) \oplus \mathcal{T}(X) \cong C_{2}(\mathcal{T}(X))$, we get $\hat{C}_{i} \in \mathscr{A}_{l, s}^{C_{0}}\left(C_{2}(\mathcal{T}(X))\right)$. Set $Y:=j_{C_{2}(X)}\left(C_{2}(X)\right)$. With the theorem of Lumer-Phillips and Proposition 3.19 it follows that $\left.\hat{C}_{i}\right|_{Y} \in \mathscr{A}_{l, s}^{C_{0}}(Y)$. We obtain i $\left(\begin{array}{cc}0 & A^{*} \\ A & 0\end{array}\right) \in \mathscr{A}_{l, s}^{C_{0}}\left(C_{2}(X)\right)$; thus $A^{*} \in \mathscr{A}_{l}^{C_{0}}(X)$ and $\left(A^{*}\right)^{*}=A$.

We can now prove the equivalence of (a) and (c) in Theorem 3.13

Let $A \in \mathscr{A}_{l}^{C_{0}}(X)$. Due to Proposition 3.18 and Theorem 3.8 there exists $B \in \mathscr{A}_{l}^{C_{0}}(\mathcal{T}(X))=\mathcal{R}(\mathcal{T}(X))$ satisfying $j \circ A=\left.B \circ j\right|_{D(A)}$. Then Theorem 3.20 implies $\pm \mathrm{i}\left(\begin{array}{cc}0 & A \\ A^{*} & 0\end{array}\right)= \pm\left.\mathrm{i}\left(\begin{array}{cc}0 & B \\ B^{*} & 0\end{array}\right)\right|_{j_{C_{2}(X)}\left(C_{2}(X)\right)} \in \mathscr{A}_{l, s}^{C_{0}}\left(C_{2}(X)\right)$, and by definition this operator generates a $C_{0}$-semigroup. With the theorem of Lumer-Phillips we obtain part (c)(ii) of Theorem 3.13 .

Conversely, suppose there exists $B \in \mathcal{R}(\mathcal{T}(X))=\mathscr{A}_{l}^{C_{0}}(\mathcal{T}(X))$ satisfying (i) and (ii) of (c). With Proposition 3.19 we conclude that $\left.B\right|_{j(X)} \in \mathscr{A}_{l}^{C_{0}}(j(X))$; thus $A \in \mathscr{A}_{l}^{C_{0}}(X)$.

3.4. Connection to operators on Hilbert spaces. In this subsection we characterize unbounded skew-adjoint multipliers using $C_{0}$-groups on Hilbert spaces.

Theorem 3.21. Let $X$ be an operator space. Let $\left(\pi, K, K_{1}, K_{2}\right)$ be a $\mathcal{T}(X)$ embedding of $X$ and let $\eta:=\pi \circ j: X \rightarrow L(K)$. Let $A: D(A) \subseteq X \rightarrow X$ be linear. 
The following assertions are equivalent:

(a) $A \in \mathscr{A}_{l, s}^{C_{0}}(X)$.

(b) $A$ is densely defined with $\rho(A) \neq \emptyset$, and there exists a $C_{0}$-group $\left(b_{t}\right)_{t \geq 0}$ of unitary elements in $L\left(K_{2}\right)$ such that:

(i) $L_{b_{t}}: \eta(X) \rightarrow \eta(X), y \mapsto b_{t} y$ and $L_{b_{t}^{*}}$ define $C_{0}$-groups on $\eta(X)$ and

(ii) for the generator $C$ of $\left(L_{b_{t}}\right)_{t \in \mathbb{R}}$, we have $j(D(A)) \subseteq D(C)$.

Proof. At first we show that (a) implies (b). Let $\left(T_{t}\right)_{t \in \mathbb{R}}$ denote the $C_{0}$-group generated by $A$. There exists $a_{t} \in \mathcal{I} M_{\ell}^{*}(X)$ such that $j\left(T_{t} x\right)=a_{t} \cdot j(x)$. Define $b_{t}:=\left.\pi\left(a_{t}\right)\right|_{K_{2}}$. Because $\left[\pi(\mathcal{T}(X)) K_{1}\right]$ is dense in $K_{2}$, we have $b_{t}\left(K_{2}\right) \subseteq K_{2}$. With equation (3.3) it follows for all $z \in \mathcal{T}(X)$ and $\xi \in K_{1}$ that

$$
\left\|b_{t}(\pi(z)(\xi))-\pi(z)(\xi)\right\|=\left\|\left(\pi\left(a_{t}\right) \pi(z)-\pi(z)\right)(\xi)\right\| \leq\left\|a_{t} \cdot z-z\right\|\|\xi\| \rightarrow 0
$$

for $t \rightarrow 0$. Therefore $\left(b_{t}\right)_{t \in \mathbb{R}}$ is a $C_{0}$-group on $K_{2}$. Using the theorem of HilleYosida, we obtain that $A$ is densely defined with $\rho(A) \neq \emptyset$. It is straightforward to show (i) and (ii).

To prove the other direction, define $d_{t}: K \rightarrow K, \xi \mapsto b_{t}\left(p_{K_{2}}(\xi)\right)$, where $p_{K_{2}}$ denotes the projection from $K$ onto $K_{2}$ and $S_{t}:=L_{d_{t}}: \eta(X) \rightarrow \eta(X)$. Then it can be checked that $T_{t}:=\eta^{-1} \circ S_{t} \circ \eta$ defines a $C_{0}$-group on $X$ with generator $A$.

With the above theorem we obtain the equivalence of (a) and (d) in Theorem 3.13. So we have finished the proof of this theorem.

Proposition 3.22. Let $X$ be an operator space and $A \in \mathscr{A}_{l, s}^{C_{0}}(X)$. There exist a Hilbert space $K$, a completely isometric map $\eta: X \rightarrow L(K)$ and a generator $C$ of a unitary $C_{0}$-group on $K$ such that $\eta(A x)=C \circ \eta(x)$ for all $x \in D(A)$.

Proof. We use the notation of Theorem 3.21. Using this result, we find a $C_{0}$-group $\left(b_{t}\right)_{t \in \mathbb{R}}$ with the properties stated in Theorem 3.21. Let $\tilde{C}$ be the generator of this $C_{0}$-group. With Theorem 3.21 we get

$$
\eta(A x)(\xi)=\lim _{t \rightarrow 0} \frac{\left(b_{t} \circ \eta(x)\right)(\xi)-\eta(x)(\xi)}{t}=\tilde{C}(\eta(x)(\xi))
$$

for all $x \in D(A)$ and $\xi \in K$; thus $\eta(A x)=\tilde{C} \circ \eta(x)$. The assertion follows from setting $C:=\tilde{C} \oplus 0_{K_{2}^{\perp}}$.

3.5. The strict $X$-topology. If we want to transform a $C_{0}$-group $\left(b_{t}\right)_{t \in \mathbb{R}}$ from a Hilbert space to an operator space $X$, we have to impose a stronger condition than strong continuity on $\left(b_{t}\right)_{t \in \mathbb{R}}$ (cf. Theorem 3.21), namely

$$
\lim _{t \rightarrow 0} b_{t} \circ y=y \quad \text { for all } y \in \eta(X) .
$$

In order to formulate this in a more abstract fashion, we introduce the following topology for an operator space $X \subseteq L\left(H_{0}, H\right)$ :

Definition 3.23. For all $x \in X$ a seminorm is defined by

$$
p_{x}: L(H) \rightarrow \mathbb{R}, T \mapsto\|T \circ x\| .
$$

The locally convex topology on $L(H)$ generated by $\left(p_{x}\right)_{x \in X}$ is called the strict $X$-topology.

The norm topology on $L(H)$ is finer than the strict $X$-topology on $L(H)$. If $X$ is unital and $H_{0}=H$, these two topologies coincide. 
The proof of the following proposition is straightforward:

Proposition 3.24. Let $X \subseteq L\left(H_{0}, H\right)$ be an operator space with $\left[X H_{0}\right]$ dense in $H$. The strict $X$-topology on $L(H)$ is finer than the strong topology.

It is not hard to see that the assumption in the above proposition can always be fulfilled by choosing an appropriate embedding of $X$.

Proposition 3.25. Let $X$ be an operator space. Let $K$ be a Hilbert space and $\eta: X \rightarrow L(K)$ a completely isometric map. Let $C: D(C) \subseteq K \rightarrow K$ be the generator of a $C_{0}$-group $\left(b_{t}\right)_{t \in \mathbb{R}}$ of unitary elements in $L(K)$ such that

(i) $\left(b_{t}\right)_{t}$ converges in the strict $\eta(X)$-topology to $\mathrm{id}_{K}$ for $t \rightarrow 0$ and

(ii) $b_{t} \eta(X), b_{t}^{*} \eta(X) \subseteq \eta(X)$ for all $t \in \mathbb{R}$.

Then there exists $A \in \mathscr{A}_{l, s}^{C_{0}}(X)$ such that

$$
\eta(A x)(\xi)=C(\eta(x)(\xi)) \quad \text { for all } x \in D(A), \xi \in K .
$$

Proof. The proof is similar to the proof of Proposition 3.22

3.6. Pertubation theory. The following result generalizes a pertubation result of Damaville ([Dam04, Proposition 2.1]; see also [Dam07]) from Hilbert $C^{*}$-modules to operator spaces:

Theorem 3.26. Let $X$ be an operator space.

(i) If $A \in \mathscr{M}_{l}^{C_{0}}(X)$ and $B \in \mathscr{M}_{l}(X)$, then $A+B \in \mathscr{M}_{l}^{C_{0}}(X)$.

(ii) If $A \in \mathscr{A}_{l, s}^{C_{0}}(X)$ and $B \in \mathscr{A}_{l}(X)$ with $B^{*}=-B$, then $A+B \in \mathscr{A}_{l, s}^{C_{0}}(X)$.

(iii) If $A \in \mathscr{A}_{l}^{C_{0}}(X)$ and $B \in \mathscr{A}_{l}(X)$, then $A+B \in \mathscr{A}_{l}^{C_{0}}(X)$.

Proof. Part (i) follows directly with [EN00, Theorem III.1.10].

To prove (ii), we notice that there exists $\check{A} \in \mathscr{A}_{l, s}^{C_{0}}(\mathcal{T}(X))=\mathcal{R}(\mathcal{T}(X))$ satisfying $j \circ A=\left.\check{A} \circ j\right|_{D(A)}$ due to Theorem 3.18 . There exists $\check{B} \in \mathscr{A}_{l}(\mathcal{T}(X)) \cong \mathbb{B}(\mathcal{T}(X))$ skew-adjoint satisfying $j \circ B=\check{B} \circ j$. Using Dam04, Proposition 2.1], we conclude that $\check{A}+\check{B} \in \mathcal{R}(\mathcal{T}(X))$. With Theorem 3.4 we get: $\check{A}+\check{B} \in \mathscr{A}_{l, s}^{C_{0}}(\mathcal{T}(X))$. Because $B$ and the $C_{0}$-group generated by $A$ leave $j(X)$ invariant, the $C_{0}$-group generated by $\check{A}+\check{B}$ leaves $j(X)$ invariant. We obtain with Sch09, Proposition 3.53: $(\check{A}+$ $\check{B})\left.\right|_{j(X)} \in \mathscr{A}_{l, s}^{C_{0}}(j(X))$; thus $A+B \in \mathscr{A}_{l, s}^{C_{0}}(X)$.

Part (iii) is a consequence of part (ii).

\section{ACKNOWLEDGMENT}

The authors thank the referee for a number of helpful suggestions.

\section{REFERENCES}

[BEZ02] David P. Blecher, Edward G. Effros, and Vrej Zarikian, One-sided M-ideals and multipliers in operator spaces. I, Pacific J. Math. 206 (2002), no. 2, 287-319. MR.1926779 (2003h:46084)

[BJ83] Saad Baaj and Pierre Julg, Théorie bivariante de Kasparov et opérateurs non bornés dans les $C^{*}{ }_{-}$modules hilbertiens, C. R. Math. Acad. Sci. Paris Sér. I Math. 296 (1983), no. 21, 875-878. MR715325 (84m:46091)

[Ble01] David P. Blecher, The Shilov boundary of an operator space and the characterization theorems, J. Funct. Anal. 182 (2001), no. 2, 280-343. MR1828796 (2002d:46049)

[BLM04] David P. Blecher and Christian Le Merdy, Operator algebras and their modulesan operator space approach, London Math. Soc. Monogr. New Ser., vol. 30, 2004. MR2111973 (2006a:46070) 
[BN11] David P. Blecher and Matthew Neal, Metric characteriziations of isometries and of unital operator spaces and systems, Proc. Amer. Math. Soc. 139 (2011), no. 3, 985998. MR2745650

[BP01] David P. Blecher and Vern I. Paulsen, Multipliers of operator spaces, and the injective envelope, Pacific J. Math. 200 (2001), no. 1, 1-17. MR.1863404 (2002k:46150)

[Con81] Alain Connes, An analogue of the Thom isomorphism for crossed products of a $C^{*}$ algebra by an action of R, Adv. Math. 39 (1981), no. 1, 31-55. MR605351 (82j:46084)

[Dam04] Stéphane Damaville, Régularité des opérateurs quadratiquements bornés dans les modules de Hilbert, Preprintreihe des SFB 478 - Geometrische Strukturen in der Mathematik des Mathematischen Instituts der Westfälischen Wilhelms-Universität Münster 323 (2004).

[Dam07] _ Régularité d'opérateurs non bornés dans les modules de Hilbert, C. R. Math. Acad. Sci. Paris 344 (2007), no. 12, 769-772. MR2340445

[EN00] Klaus-Jochen Engel and Rainer Nagel, One-parameter semigroups for linear evolution equations, Grad. Texts in Math., vol. 194, Springer, 2000. MR 1721989 (2000i:47075)

[ER00] Edward G. Effros and Zhong-Jin Ruan, Operator spaces, London Math. Soc. Monogr. New Ser., vol. 23, 2000. MR1793753 (2002a:46082)

[Har81] Lawrence A. Harris, A generalization of $C^{*}$-algebras, Proc. Lond. Math. Soc. (3) 42 (1981), no. 2, 331-361. MR607306 (82e:46089)

[HN12] Xu-Jian Huang and Chi-Keung Ng, An abstract characterization of unital operator spaces, J. Operator Theory 67 (2012), no. 1, 289-298. MR.2881544

[HQVK92] J. Hollevoet, J. Quaegebeur, and S. Van Keer, Stone's theorem in $C^{*}$-algebras, Q. J. Math. Oxford Ser. (2) 43 (1992), no. 170, 227-233. MR.1164625 (94e:46103)

[Kus97] Johan Kustermans, The functional calculus of regular operators on Hilbert ${ }^{*}{ }^{*}$-modules revisited, Preprint (arXiv:funct-an/9706007v1), 1997.

[Lan95] E. Christopher Lance, Hilbert $C^{*}$-modules: a toolkit for operator algebraists, London Math. Soc. Lecture Note Ser., vol. 210, 1995. MR.1325694 (96k:46100)

[Pau02] Vern Paulsen, Completely bounded maps and operator algebras, Cambridge Stud. Adv. Math., vol. 78, 2002. MR.1976867 (2004c:46118)

[Pis03] Gilles Pisier, Introduction to operator space theory, London Math. Soc. Lecture Note Ser., vol. 294, 2003. MR2006539 (2004k:46097)

[RW98] Iain Raeburn and Dana P. Williams, Morita equivalence and continuous-trace $C^{*}$ algebras, Math. Surveys Monogr., vol. 60, Amer. Math. Soc., 1998. MR.1634408 (2000c:46108)

[Sch09] Hendrik Schlieter, Unbeschränkte Multiplikatoren auf Operatorräumen, Dissertation, Westfälische Wilhelms-Universität Münster (arXiv:1007.3978v1), 2009.

[Wer99] Wend Werner, Small K-groups for operator systems, unpublished manuscript, 1999.

[Wer04] _ Multipliers on matrix ordered operator spaces and some K-groups, J. Funct. Anal. 206 (2004), no. 2, 356-378. MR2021851 (2004k:46098)

[Wer07] Dirk Werner, Funktionalanalysis, sixth edition, Springer-Verlag, Berlin, 2007.

[WN92] Stanisław L. Woronowicz and Kazimierz Napiórkowski, Operator theory in the $C^{*}$ algebra framework, Rep. Math. Phys. 31 (1992), no. 3, 353-371. MR.1232646 (94k:46123)

[Wor91] Stanisław L. Woronowicz, Unbounded elements affiliated with $C^{*}$-algebras and noncompact quantum groups, Comm. Math. Phys. 136 (1991), no. 2, 399-432. MR.1096123 (92b:46117)

Mathematisches Institut, Westfälische Wilhelms-Universität Münster, EinsteinStrasse 62, D-48149 Münster, Germany

E-mail address: hschlieter@uni-muenster.de

Mathematisches Institut, Westfälische Wilhelms-Universität Münster, EinsteinStrasse 62, D-48149 Münster, Germany

E-mail address: wwerner@math.uni-muenster.de 